\title{
ON VASSILIEV INVARIANTS FOR LINKS IN HANDLEBODIES
}

\author{
V. V. VERSHININ
}

\begin{abstract}
The notion of Vassiliev algebra in case of hanlebodies is developed. The analogues of the results of John Baez for links in handlebodies are proved. That means that there exists a one-to-one correspondence between the special class of finite type invariants of links in hanlebodies and the homogeneous Markov traces on Vassiliev algebras. This approach uses the singular braid monoid and braid group in a handlebody and the generalizations of the theorem of J. Alexander and the theorem of A. A. Markov for singular links and braids and the relative version of Markov's theorem.
\end{abstract}

\section{$\S 1$. Introduction}

Knot and link invariants introduced by V. A. Vassiliev [1] attracted a lot of attention and are studied deeply from various points of view as well as their connections with other fields of mathematics. In spite of the short period of time there exists a big amount of works on this subject.

The conception of finite type invariants was applied also for study of knots and links in 3-manifolds. Here Xiao-Song Lin [2] considered contractible manifolds and homological spheres and proved that the module of Vassiliev invariants of the order $n$ for such a manifold coincides wih that of the standard 3-dimensional euclidean space. V. A. Vassiliev continues his original approach considering the space of smooth maps from a circle to a given manifold, its discriminant, cohomology and spectral sequence [3]. V. Gorynov in his work [4] in particular develops the formalism of chord diagrams for solid torus and proves the analogue of M. Kontsevich theorem in this case.

The classical theorems of J. Alexander and A. A. Markov established the close interconnections between the study of links and the theory of braids. For Vassiliev invariants relations between singular braids and singular knots arise. They were studied by John Baez [5] and Joan Birman [6].

The aim of this paper is to carry the results of John Baez [5] for links in handlebodies. In our approach we use the braid group in a handlebody, which was studied by A. B. Sossinsky [7] and the author $[8,9]$. The essential ingredients are the generalizations of the theorem of J. Alexander and the theorem of A. A. Markov for singular links and braids proved by Joan Birman [6] and Bernd Gemein [10] and the relative version of Markov's theorem proved by Sofia Lambropoulou and Colin

1991 Mathematics Subject Classification. Primary 57M25, 20F36, 20 F38.

Key words and phrases. Vassiliev invariants, links, braid group, Markov trace, singular braid monoid, handlebody.

The research was supported in part by RFFR Grants 96-01-016331 and 96-01-00062G

Typeset by $\mathcal{A} \mathcal{M} \mathcal{S}-\mathrm{T}_{\mathrm{E}} \mathrm{X}$ 
Rourke [11]. This version allows us to have Markov's theorem for links in hanlebodies. The proofs of John Baez [5] remain valid after necessary adaptations to the case of a handlebody.

The author is thankful to Sofia Lambropoulou, Bernd Gemein and Viktor Vassiliev for supplying him with the preprints of their papers.

\section{$\S 2$. Singular braids in handlebodies}

Let $S B_{n}$ be the singular braid monoid on $n$ strings $[5,6]$, which is also called the Baez-Birman monoid or generalized braid monoid. It has the generators $\bar{\sigma}_{i}, \bar{\sigma}_{i}^{-1}$, $\bar{a}_{i}, i=1, \ldots, n-1$, and relations

$$
\begin{aligned}
& \bar{\sigma}_{i} \bar{\sigma}_{j}=\bar{\sigma}_{j} \bar{\sigma}_{i}, \text { if }|i-j|>1, \\
& \bar{a}_{i} \bar{a}_{j}=\bar{a}_{j} \bar{a}_{i}, \text { if }|i-j|>1, \\
& \bar{a}_{i} \bar{\sigma}_{j}=\bar{\sigma}_{j} \bar{a}_{i}, \text { if }|i-j| \neq 1, \\
& \bar{\sigma}_{i} \bar{\sigma}_{i+1} \bar{\sigma}_{i}=\bar{\sigma}_{i+1} \bar{\sigma}_{i} \bar{\sigma}_{i+1}, \\
& \bar{\sigma}_{i} \bar{\sigma}_{i+1} \bar{a}_{i}=\bar{a}_{i+1} \bar{\sigma}_{i} \bar{\sigma}_{i+1}, \\
& \bar{\sigma}_{i+1} \bar{\sigma}_{i} \bar{a}_{i+1}=\bar{a}_{i} \bar{\sigma}_{i+1} \bar{\sigma}_{i}, \\
& \bar{\sigma}_{i} \bar{\sigma}_{i}^{-1}=\bar{\sigma}_{i}^{-1} \bar{\sigma}_{i}=1 .
\end{aligned}
$$

Geometrically $\bar{\sigma}_{i}$ corresponds to the canonical generator of the braid group (righthanded crossing) and $\bar{a}_{i}$ represents an intersection of the $i$ th and $(i+1)$ st strand, just as in Figures 1 and 2. More detailed geometric interpretation of the Baez-Birman monoid can be found in the paper of Joan Birman [6].

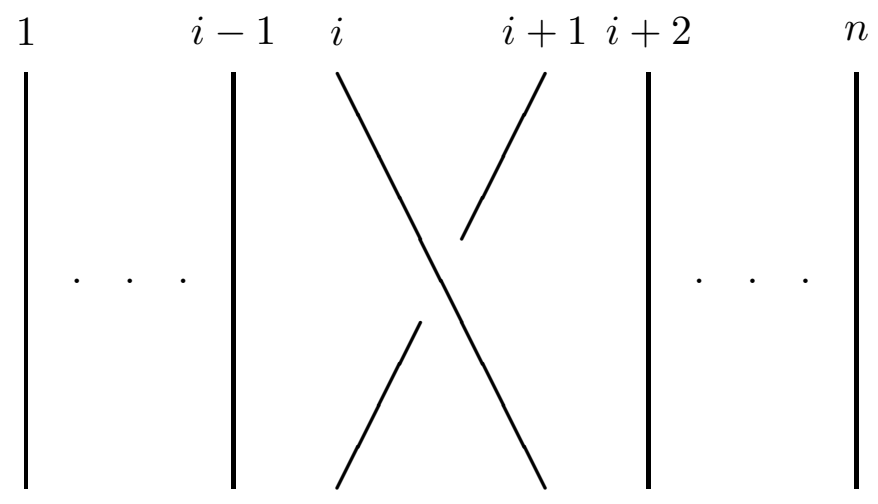

Fig. 1 


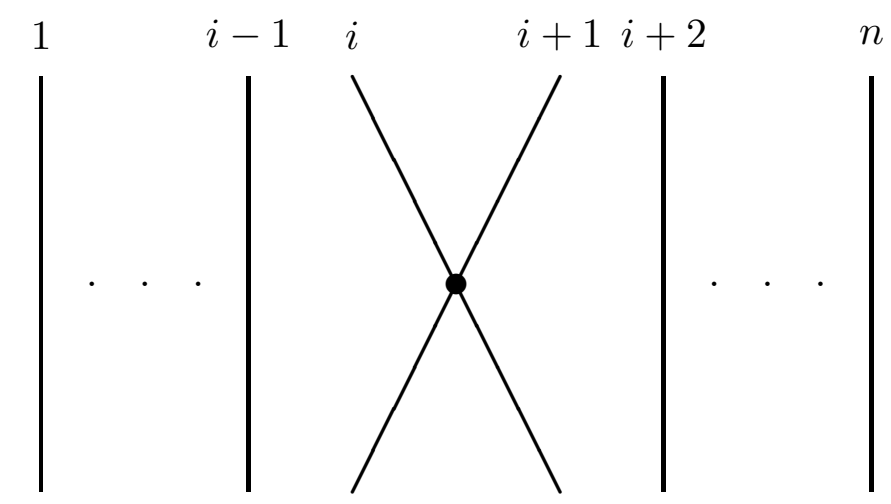

Fig. 2

As the analogous object in a handlebody of the genus $g$ it is natural to consider the submonoid of $S B_{g+n}$ in which the first $g$ strings are unbraided and noncrossing. We denote it by $S B_{n}^{g}$. The braid group $B r_{n}^{g}$ in a handlebody of the genus $g$ was studied by A. B. Sossinsky [7] and the author [8, 9]. It has the generators $\tau_{k}, k=1,2, \ldots, g$ and $\sigma_{1}, \ldots, \sigma_{n-1}$ and relations

$$
\begin{cases}\sigma_{i} \sigma_{j} & =\sigma_{j} \sigma_{i} \text { if }|i-j|>1 \\ \sigma_{i} \sigma_{i+1} \sigma_{i} & =\sigma_{i+1} \sigma_{i} \sigma_{i+1} \\ \tau_{k} \sigma_{i} & =\sigma_{i} \tau_{k} \text { if } k \geq 1, i \geq 2 \\ \tau_{k} \sigma_{1} \tau_{k} \sigma_{1} & =\sigma_{1} \tau_{k} \sigma_{1} \tau_{k}, k=1,2, \ldots, g \\ \tau_{k} \sigma_{1}^{-1} \tau_{k+l} \sigma_{1} & =\sigma_{1}^{-1} \tau_{k+l} \sigma_{1} \tau_{k}, k=1,2, \ldots, g-1 ; l=1,2, \ldots, g-k\end{cases}
$$

The group $B r_{n}^{g}$ is considered as a subgroup of the classical braid group $B r_{g+n}$ on $g+n$ strings, such that the braids from $B r_{n}^{g}$ leave the first $g$ strings unbraided. We denote also by $\bar{\sigma}_{j}$ the standard generators of the group $B r_{g+n}$. Then $\tau_{k}, k=$ $1,2, \ldots, g$, are the following braids:

$$
\tau_{k}=\bar{\sigma}_{g} \bar{\sigma}_{g-1} \ldots \bar{\sigma}_{k+1} \bar{\sigma}_{k}^{2} \bar{\sigma}_{k+1}^{-1} \ldots \bar{\sigma}_{g-1}^{-1} \bar{\sigma}_{g}^{-1}
$$

Geometrically such a braid (for $k=1$ ) is depicted in the Figure 3.

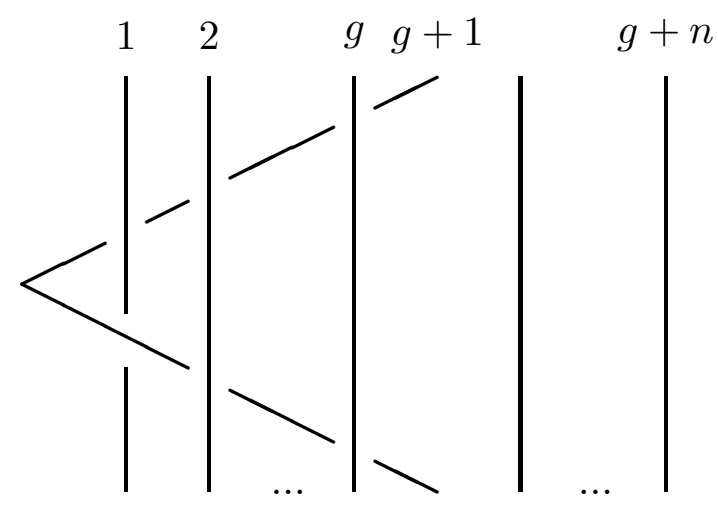

Fig. 3 
The elements $\sigma_{i} \in B r_{n}^{g}$ correspond to $\bar{\sigma}_{i+g}$.

The monoid $S B_{n}^{g}$ can be considered as a submonoid of $S B_{g+n}$ generated by the elements $\sigma_{i}=\bar{\sigma}_{i+g}, \tau_{k}, k=1,2, \ldots, g$ and $a_{i}=\bar{a}_{i+g}$.

It is proved by R. Fenn, E. Keyman and C. Rourke [12] that the Baez-Birman monoid $S B_{k}$ embeds in a group $S G_{k}$ which they call the singular braid group:

$$
S B_{n} \rightarrow S G_{n}
$$

It means that the elements $\bar{a}_{i}$ become invertible and all the relations of $S B_{k}$ remain true.

It seems to be rather complicated to write a presentation of monoid $S B_{n}^{g}$, but some relations can be obtained easily. Let us introduce the following notations:

$$
\tau_{i, m}=\tau_{i} \tau_{i+1} \ldots \tau_{m}, \quad i \leq m
$$

Proposition 1. The following relations are fulfilled in the monoid $S B_{g+n}$ and hence in the group $S G_{g+n}$ :

$$
\tau_{i, g} \bar{\sigma}_{g+1} \tau_{i, g} \bar{a}_{g+1}=\bar{a}_{g+1} \tau_{i, g} \bar{\sigma}_{g+1} \tau_{i, g}, \quad i \leq g .
$$

Proof. These relations are obtained in the following way. We express the elements $\bar{a}_{i}, i \leq g$, by means of $\bar{a}_{g+1}$ and $\bar{\sigma}_{j}$ using firstly the fifth relation in (1) and secondly the sixth relation in (1). Then we equate these two expressions. We start with $\bar{a}_{g}$ :

$$
\begin{aligned}
& \bar{a}_{g}=\bar{\sigma}_{g+1} \bar{\sigma}_{g} \bar{a}_{g+1} \bar{\sigma}_{g}^{-1} \bar{\sigma}_{g+1}^{-1}, \\
& \bar{a}_{g}=\bar{\sigma}_{g+1}^{-1} \bar{\sigma}_{g}^{-1} \bar{a}_{g+1} \bar{\sigma}_{g} \bar{\sigma}_{g+1} .
\end{aligned}
$$

Hence we have

$$
\bar{\sigma}_{g} \bar{\sigma}_{g+1}^{2} \bar{\sigma}_{g} \bar{a}_{g+1}=\bar{a}_{g+1} \bar{\sigma}_{g} \bar{\sigma}_{g+1}^{2} \bar{\sigma}_{g}
$$

Let us multiply on the right side both parts of the equality (3) by $\bar{\sigma}_{g+1}$ and then use the braid group relations and the relation $\bar{\sigma}_{g+1} \bar{a}_{g+1}=\bar{a}_{g+1} \bar{\sigma}_{g+1}$. We get the following equalities for the left part of (3):

$$
\bar{\sigma}_{g+1} \bar{\sigma}_{g} \bar{\sigma}_{g+1}^{2} \bar{\sigma}_{g} \bar{a}_{g+1}=\bar{\sigma}_{g} \bar{\sigma}_{g+1} \bar{\sigma}_{g} \bar{\sigma}_{g+1} \bar{\sigma}_{g} \bar{a}_{g+1}=\bar{\sigma}_{g}^{2} \bar{\sigma}_{g+1} \bar{\sigma}_{g}^{2} \bar{a}_{g+1}
$$

and analogously for the right part:

$$
\bar{\sigma}_{g+1} \bar{a}_{g+1} \bar{\sigma}_{g} \bar{\sigma}_{g+1}^{2} \bar{\sigma}_{g}=\bar{a}_{g+1} \bar{\sigma}_{g}^{2} \bar{\sigma}_{g+1} \bar{\sigma}_{g}^{2}
$$

Using the expressions for $\tau_{k}$ we obtain:

$$
\tau_{g, g} \bar{\sigma}_{g+1} \tau_{g, g} \bar{a}_{g+1}=\bar{a}_{g+1} \tau_{g, g} \bar{\sigma}_{g+1} \tau_{g, g} .
$$

In general case we have the following expressions

$$
\begin{gathered}
\bar{a}_{1}=\bar{\sigma}_{2} \bar{\sigma}_{1} \bar{\sigma}_{3} \bar{\sigma}_{2} \ldots \bar{\sigma}_{g} \bar{a}_{g+1} \bar{\sigma}_{g}^{-1} \ldots \bar{\sigma}_{2}^{-1} \bar{\sigma}_{3}^{-1} \bar{\sigma}_{1}^{-1} \bar{\sigma}_{2}^{-1}, \\
\bar{a}_{2}=\bar{\sigma}_{3} \bar{\sigma}_{2} \ldots \bar{\sigma}_{g} \bar{a}_{g+1} \bar{\sigma}_{g}^{-1} \ldots \bar{\sigma}_{2}^{-1} \bar{\sigma}_{3}^{-1}
\end{gathered}
$$




$$
\begin{gathered}
\bar{a}_{i}=\bar{\sigma}_{i+1} \bar{\sigma}_{i} \bar{\sigma}_{i+2} \bar{\sigma}_{i+1} \ldots \bar{\sigma}_{g} \bar{a}_{g+1} \bar{\sigma}_{g}^{-1} \ldots \bar{\sigma}_{i+1}^{-1} \bar{\sigma}_{i+2}^{-1} \bar{\sigma}_{i}^{-1} \bar{\sigma}_{i+1}^{-1}, \\
\ldots, \\
\bar{a}_{g-1}=\bar{\sigma}_{g} \bar{\sigma}_{g-1} \bar{\sigma}_{g+1} \bar{\sigma}_{g} \bar{a}_{g+1} \bar{\sigma}_{g}^{-1} \bar{\sigma}_{g+1}^{-1} \bar{\sigma}_{g-1}^{-1} \bar{\sigma}_{g}^{-1}, \\
\bar{a}_{1}=\bar{\sigma}_{2}^{-1} \bar{\sigma}_{1}^{-1} \bar{\sigma}_{3}^{-1} \bar{\sigma}_{2}^{-1} \ldots \bar{\sigma}_{g}^{-1} \bar{a}_{g+1} \bar{\sigma}_{g} \ldots \bar{\sigma}_{2} \bar{\sigma}_{3} \bar{\sigma}_{1} \bar{\sigma}_{2}, \\
\bar{a}_{2}=\bar{\sigma}_{3}^{-1} \bar{\sigma}_{2}^{-1} \ldots \bar{\sigma}_{g}^{-1} \bar{a}_{g+1} \bar{\sigma}_{g} \ldots \bar{\sigma}_{2} \bar{\sigma}_{3}, \\
\ldots, \\
\bar{a}_{i}=\bar{\sigma}_{i+1}^{-1} \bar{\sigma}_{i}^{-1} \bar{\sigma}_{i+2}^{-1} \bar{\sigma}_{i+1}^{-1} \ldots \bar{\sigma}_{g}^{-1} \bar{a}_{g+1} \bar{\sigma}_{g} \ldots \bar{\sigma}_{i+1} \sigma_{i+2} \sigma_{i} \sigma_{i+1}, \\
\ldots, \\
\bar{a}_{g-1}=\bar{\sigma}_{g}^{-1} \bar{\sigma}_{g-1}^{-1} \bar{\sigma}_{g+1}^{-1} \bar{\sigma}_{g}^{-1} \bar{a}_{g+1} \bar{\sigma}_{g} \bar{\sigma}_{g+1} \bar{\sigma}_{g-1} \bar{\sigma}_{g},
\end{gathered}
$$

From the equations (4) and (5) we get

$$
\bar{\sigma}_{g} \bar{\sigma}_{g+1} \ldots \bar{\sigma}_{i} \bar{\sigma}_{i+1}^{2} \bar{\sigma}_{i} \ldots \bar{\sigma}_{g+1} \bar{\sigma}_{g} \bar{a}_{g+1}=\bar{a}_{g+1} \bar{\sigma}_{g} \bar{\sigma}_{g+1} \ldots \bar{\sigma}_{i} \bar{\sigma}_{i+1}^{2} \bar{\sigma}_{i} \ldots \bar{\sigma}_{g+1} \bar{\sigma}_{g}
$$

We multiply on the right side both parts of the equality (6) by $\bar{\sigma}_{g+1}$ and then use the braid group relations and the relation $\bar{\sigma}_{g+1} \bar{a}_{g+1}=\bar{a}_{g+1} \bar{\sigma}_{g+1}$. We have the following equalities for the left part of (6):

$$
\begin{gathered}
\bar{\sigma}_{g+1} \bar{\sigma}_{g} \bar{\sigma}_{g+1} \ldots \bar{\sigma}_{i} \bar{\sigma}_{i+1}^{2} \bar{\sigma}_{i} \ldots \bar{\sigma}_{g+1} \bar{\sigma}_{g} \bar{a}_{g+1}=\bar{\sigma}_{g} \bar{\sigma}_{g+1} \bar{\sigma}_{g} \ldots \bar{\sigma}_{i} \bar{\sigma}_{i+1}^{2} \bar{\sigma}_{i} \ldots \bar{\sigma}_{g+1} \bar{\sigma}_{g} \bar{a}_{g+1}=\ldots \\
=\bar{\sigma}_{g} \bar{\sigma}_{g+1} \bar{\sigma}_{g} \ldots \bar{\sigma}_{i+1} \bar{\sigma}_{i} \bar{\sigma}_{i+1}^{2} \bar{\sigma}_{i} \ldots \bar{\sigma}_{g+1} \bar{\sigma}_{g} \bar{a}_{g+1}= \\
=\bar{\sigma}_{g} \bar{\sigma}_{g+1} \bar{\sigma}_{g} \ldots \bar{\sigma}_{i+2} \bar{\sigma}_{i}^{2} \bar{\sigma}_{i+1} \bar{\sigma}_{i}^{2} \bar{\sigma}_{i+2} \ldots \bar{\sigma}_{g+1} \bar{\sigma}_{g} \bar{a}_{g+1}= \\
=\bar{\sigma}_{g} \bar{\sigma}_{g+1} \bar{\sigma}_{g} \ldots \bar{\sigma}_{i}^{2} \bar{\sigma}_{i+1} \bar{\sigma}_{i+2} \bar{\sigma}_{i+1} \bar{\sigma}_{i}^{2} \ldots \bar{\sigma}_{g+1} \bar{\sigma}_{g} \bar{a}_{g+1}=\ldots= \\
=\bar{\sigma}_{g} \bar{\sigma}_{g-1} \ldots \bar{\sigma}_{i}^{2} \bar{\sigma}_{i+1} \ldots \bar{\sigma}_{g} \bar{\sigma}_{g+1} \bar{\sigma}_{g} \ldots \bar{\sigma}_{i}^{2} \bar{\sigma}_{i+1} \ldots \bar{\sigma}_{g}=\tau_{i} \tau_{i+1} \ldots \tau_{g} \bar{\sigma}_{g+1} \tau_{i} \tau_{i+1} \ldots \tau_{g} \bar{a}_{g+1} .
\end{gathered}
$$

Analogous transformations for the right side of (6) give the equation

$$
\tau_{i, g} \bar{\sigma}_{g+1} \tau_{i, g} \bar{a}_{g+1}=\bar{a}_{g+1} \tau_{i, g} \bar{\sigma}_{g+1} \tau_{i, g}
$$

Corollary 1. The following relations are fulfilled in the monoid $S B_{n}^{g}$ :

$$
\tau_{i, g} \sigma_{1} \tau_{i, g} a_{1}=a_{1} \tau_{i, g} \sigma_{1} \tau_{i, g}, \quad i \leq g
$$

Remark. The analogous relations are fulfilled in the braid group $B r_{g+n}$ and hence in the braid group in a handlebody $\mathrm{Br}_{n}^{g}$ :

$$
\tau_{i, m} \bar{\sigma}_{m+1} \tau_{i, m} \bar{\sigma}_{m+1}=\bar{\sigma}_{m+1} \tau_{i, m} \bar{\sigma}_{m+1}, \quad i \leq m
$$

The proof is straightforward using the induction on $m-i$. 
There exist maps which are right inverses of the homomorphism of the canonical inclusion

$$
j_{k}: B r_{k} \rightarrow S G_{k}
$$

One of them

$$
h_{k}: S G_{k} \rightarrow B r_{k},
$$

is defined by the formulas:

$$
\begin{gathered}
h_{k}\left(\bar{\sigma}_{i}\right)=\bar{\sigma}_{i}, \\
h_{k}\left(\bar{a}_{i}\right)=e,
\end{gathered}
$$

and another one

$$
h_{k}^{\prime}: S G_{k} \rightarrow B r_{k},
$$

by the following action on generators:

$$
\begin{aligned}
& h_{k}^{\prime}\left(\bar{\sigma}_{i}\right)=\bar{\sigma}_{i}, \\
& h_{k}^{\prime}\left(\bar{a}_{i}\right)=\bar{\sigma}_{i} .
\end{aligned}
$$

The image of the composition

$$
S G_{n}^{g} \stackrel{\text { Incl }}{\longrightarrow} S G_{g+n} \stackrel{h_{g+n}}{\longrightarrow} B r_{g+n}
$$

lies in $B r_{n}^{g}$. We define a homomorphism $h_{n}^{g}$ to make the following diagram commutative

$$
\begin{array}{rlr}
S G_{n}^{g} \stackrel{\text { Incl }}{\longrightarrow} & S G_{g+n} \\
\downarrow h_{n}^{g} & \\
& \downarrow h_{g+n} \\
B r_{n}^{g} \stackrel{\text { Incl }}{\longrightarrow} & B r_{g+n} .
\end{array}
$$

These homomorphisms fit for the following commutative diagram

$$
\begin{aligned}
& S G_{n} \stackrel{\text { Incl }}{\longrightarrow} S G_{n}^{g} \stackrel{\text { Incl }}{\longrightarrow} S G_{g+n} \\
& \downarrow h_{n} \quad \downarrow h_{n}^{g} \quad \downarrow h_{g+n} \\
& B r_{n} \stackrel{\text { Incl }}{\longrightarrow} B r_{n}^{g} \stackrel{\text { Incl }}{\longrightarrow} B r_{g+n} \text {. }
\end{aligned}
$$

Let $\operatorname{deg}_{k}$ be the homomorphism

$$
S G_{k} \rightarrow \mathbb{Z}
$$

which assigns to each element of the group $S G_{k}$ the sum of degrees of the generators $\bar{a}_{i}$, which occur in this element. We define a homomorphism

$$
\operatorname{deg}_{n}^{g}: S G_{n}^{g} \rightarrow \mathbb{Z}
$$

as the following composition

$$
S G_{n}^{g} \stackrel{\text { Incl }}{\longrightarrow} S G_{g+n} \stackrel{\operatorname{deg}_{n}^{g}}{\longrightarrow} \mathbb{Z} .
$$

One defines a homomorphism

$$
\mathbb{Z} \rightarrow S G_{n}^{g}
$$

as an inclusion of the cyclic group as a subgroup generated by the element $a_{1}$. Then the composition

$$
\mathbb{Z} \rightarrow S G_{n}^{g} \stackrel{\operatorname{deg}_{n}^{g}}{\longrightarrow} \mathbb{Z}
$$

is equal to identity. 


\section{$\S 3$. Vassiliev algebra for handlebodies}

Let $K$ be a commutative ring with unit, $K[\epsilon]$ is a polynomial ring on one variable $\epsilon$ over $K, K S B_{n}^{g}$ denotes the semigroup algebra of the monoid (semigroup with the unit) $S B_{n}^{g}$ over $K$.

Definition 1. We call Vassiliev algebra $V_{n}^{g}$ the factor-algebra of $K S B_{n}^{g} \otimes K[\epsilon]$ by the ideal generated by the relations

$$
\sigma_{i}-\sigma_{i}^{-1}=\epsilon a_{i}
$$

Remark. Definitions and results of the paper of J. Baez [5] follow if we take $g=0$. We omit writing zero in these cases.

The homomorphism of inclusion

$$
S B_{n}^{g} \rightarrow S B_{g+n}
$$

induces the homomorphism of algebras:

$$
\alpha: V_{n}^{g} \rightarrow V_{n+g}
$$

such that $\alpha\left(\sigma_{i}\right)=\bar{\sigma}_{g+i}, \alpha\left(a_{i}\right)=\bar{a}_{g+i}, \alpha\left(\tau_{k}\right)=\bar{\sigma}_{g} \bar{\sigma}_{g-1} \ldots \bar{\sigma}_{k+1} \bar{\sigma}_{k}^{2} \bar{\sigma}_{k+1}^{-1} \ldots \bar{\sigma}_{g-1}^{-1} \bar{\sigma}_{g}^{-1}$.

We define the homomorphism

$$
v^{g}: K B r_{n}^{g} \rightarrow V_{n}^{g}
$$

by the formulas:

$$
\begin{gathered}
v^{g}\left(\sigma_{i}\right)=\sigma_{i}, \\
v^{g}\left(\tau_{i}\right)=\tau_{i} .
\end{gathered}
$$

Let $K(\epsilon)$ be the algebra of Laurent polynomials in $\epsilon$ over $K$.

Proposition 2. The homomorphism

$$
v^{g} \otimes 1: K B r_{n}^{g} \otimes K(\epsilon) \rightarrow V_{n}^{g} \otimes_{K[\epsilon]} K(\epsilon)
$$

is an isomorphism.

Proof. The generators of $V_{n}^{g} \otimes_{K[\epsilon]} K(\epsilon)$ are the images of the elements from $K B r_{n}^{g} \otimes$ $K(\epsilon)$ : for $\sigma_{i}$ and $\tau_{i}$ this follows from definition and for $a_{j}$ from the formula

$$
\left(v^{g} \otimes 1\right)\left(\left(\sigma_{i}-\sigma_{i}^{-1}\right) \otimes \epsilon^{-1}\right)=a_{j} \otimes 1 .
$$

So $v^{g} \otimes 1$ is an epimorphism. Let us consider the commutative diagram

$$
\begin{array}{cc}
K B r_{n}^{g} \otimes K(\epsilon) \stackrel{v^{g} \otimes 1}{\longrightarrow} & V_{n}^{g} \otimes_{K[\epsilon]} K(\epsilon) \\
\downarrow \text { Incl } \otimes 1 & \downarrow \alpha \\
K B r_{n+g} \otimes K(\epsilon) \stackrel{v \otimes 1}{\longrightarrow} & V_{n+g} \otimes_{K[\epsilon]} K(\epsilon) .
\end{array}
$$

The homomorphism Incl $\otimes 1$ is a monomorphism, because all the modules are free. The homomorphism $v \otimes 1$ is a monomorphism, because it has evidently defined right inverse. Hence $v^{g} \otimes 1$ is a monomorphism and then an isomorphism.

Remark. This Proposition is more important in the case of a handlebody than in the classical case because the presentation of the Vassiliev algebra in this case is not so evident. 


\section{$\S 4$. Invariants of links in handlebodies}

Let $\mathcal{L}$ be a $K$-valued link invariant by what we mean an ambient isotopy invariant of oriented links in a handlebody of a genus $g$. It uniquely extends to a $K(\epsilon)$-valued invariant of generalized links admitting transverse double points by the standard procedure

$$
\mathcal{L}\left(L_{+}\right)-\mathcal{L}\left(L_{-}\right)=\epsilon \mathcal{L}\left(L_{\times}\right)
$$

where $L_{+}, L_{-}$and $L_{\times}$denote link diagrams with a right handed crossing, left handed crossing, and an intersection respectively, at a given point, the rest of the diagram being the same. Usually $\epsilon$ is supposed to be equal to 1 in this equation. An invariant $\mathcal{L}$ is called to be of degree $d$ if it vanishes on all generalized links with at least $d+1$ self-intersections. A link invariant of degree $d$ for some $d$ is said to be of finite type.

For all $n$ there are inclusions $V_{n}^{g} \subset V_{n+1}^{g}$ and $K B r_{n}^{g} \subset K B r_{n+1}^{g}$. We consider the direct limits $V_{\infty}^{g}$ and $K B r_{\infty}^{g}$ and omit index $\infty$ in notations.

Definition 2. We call a Markov trace on $V^{g}$ to be a $K[\epsilon]$-linear homomorphism to some $K[\epsilon]$-module $E$

$$
T: V^{g} \rightarrow E,
$$

satisfying the following conditions

$$
\begin{gathered}
T(x y)=T(y x) \text { for all } x, y \in V^{g}, \\
T\left(x \sigma_{n}^{ \pm 1}\right)=z T(x) \text { for some } z \in K \text { and for all } x \in V_{n}^{g} \subset V^{g} .
\end{gathered}
$$

Similarly is defined the Markov trace to some $K$-module $M$

$$
\operatorname{tr}: \mathrm{KBr}^{g} \rightarrow M
$$

This definition of Markov traces is analogous to the classical one for usual braids

$$
\operatorname{tr}: K B r \rightarrow M
$$

Definition 3. A Markov trace

$$
T: V^{g} \rightarrow K[\epsilon]
$$

is said to be homogeneous of degree $d$ if for every $x \in K B r^{g}$ its image $T(v(x)) \in K[\epsilon]$ is a homogeneous polynomial on $\epsilon$ of degree $d$.

For a braid $x \in B r_{n}^{g}$ we denote by $\hat{x}$ its closure with respect to the last $n$ strings. It is considered as a link in a handlebody of the genus $g$. For a link $L$ we denote by $L \cup \circ$ the unlinked union with the unknot (which is also supposed to be unlinked with the holes of the handlebody).

Theorem 1. There is a one-to-one correspondence between $K$-valued link invariants $\mathcal{L}$ of degree $d$ such that for some $z \in K^{*}$ (group of units of the ring $K$ )

$$
z \mathcal{L}(L \cup \circ)=\mathcal{L}(L)
$$

and Markov traces

$$
T: V^{g} \rightarrow K[\epsilon]
$$


that are homogeneous of degree $d$. The invariant $\mathcal{L}$ determines the trace $T$, and conversely by the formula

$$
T\left(v^{g}(x)\right)=\epsilon^{d} z^{n-1} \mathcal{L}(\hat{x}), \text { for } x \in B r_{n}^{g} .
$$

Proof. Let $M$ be $K$-module, $\mathcal{L}$ is a $M$-valued link invariant, satisfying the equation (7). Then by the relative version of the Markov's theorem proved by Sofia Lambropoulou and Colin Rourke [11, Theorem 4.7] there exists a Markov trace

$$
\operatorname{tr}: K B r^{g} \rightarrow M,
$$

given by the formula

$$
\operatorname{tr}(x)=z^{n-1} \mathcal{L}(\hat{x}), \text { for all } x \in B r_{n}^{g} .
$$

We note that this trace is well-defined because if $y \in K B r_{n+1}^{g}$ is the image of $x \in B r_{n}^{g}$ under the canonical inclusion $K B r_{n}^{g} \subset K B r_{n+1}^{g}$, then

$$
\operatorname{tr}(y)=z^{n} \mathcal{L}(\hat{y})=z^{n} \mathcal{L}(\hat{x} \cup \circ)=z^{n-1} \mathcal{L}(\hat{x})=\operatorname{tr}(x) .
$$

Moreover, $t r$ is a trace because $\widehat{x y}=\widehat{y x}$ for all $x, y \in B r_{n}^{g}$. We also have

$$
\operatorname{tr}\left(x \sigma_{n}^{ \pm 1}\right)=z^{n} \mathcal{L}\left(\widehat{x \sigma_{n}^{ \pm 1}}\right)=z^{n} \mathcal{L}(\hat{x})=z \operatorname{tr}(x) .
$$

So, equation (9) gives a one-to-one correspondence between $M$-valued link invariants satisfying (7) and Markov traces

$$
\operatorname{tr}: K B r^{g} \rightarrow M
$$

Now let $\mathcal{L}$ be $K$-valued link invariant of degree $d$ satisfying equation (7). Then there is a unique Markov trace

$$
\operatorname{tr}: K B r^{g} \rightarrow K
$$

given by the equation (9). Then by the Proposition 2 there exists a map

$$
\tilde{T}: V^{g} \otimes_{K[\epsilon]} K(\epsilon) \rightarrow K(\epsilon),
$$

such that

$$
\tilde{T}(v \otimes 1)=\epsilon^{d}(\operatorname{tr} \otimes 1): K B r_{n}^{g} \otimes K(\epsilon) \rightarrow K(\epsilon) .
$$

Let $T_{0}$ be defined as the composition

$$
V^{g} \cong V^{g} \otimes_{K[\epsilon]} K[\epsilon] \stackrel{1 \otimes \text { Incl }}{\longrightarrow} V^{g} \otimes_{K[\epsilon]} K(\epsilon) \stackrel{\tilde{T}}{\longrightarrow} K(\epsilon) .
$$

It follows that

$$
T_{0}(v(x))=\epsilon^{d} \operatorname{tr}(x), \quad x \in K B r^{g} .
$$


If an element $x \in V^{g}$ can be written as a product of $l$ generators $a_{i}$ and an arbitrary number of the generators $\sigma_{j}$ or $\tau_{k}$, then $T_{0}(x)=c \epsilon^{d-l}$ for $c \in K$. Moreover, if $l>d$, then $T_{0}(x)=0$, since $\mathcal{L}$ is invariant of degree $d$. Hence

$$
T_{0}(x)=\sum_{i=0}^{d} c_{i} \epsilon^{i}, \quad c_{i} \in K
$$

for arbitrary $x \in V^{g}$. This means that $T_{0}$ factors through a map

$$
T: V^{g} \rightarrow K[\epsilon]
$$

It is easy to check that $T$ satisfies necessary conditions.

Conversely, suppose that $T: V^{g} \rightarrow K[\epsilon]$ is a homogeneous Markov trace of degree $d$. We define a Markov trace $\operatorname{tr}: K B r^{g} \rightarrow K$ by the formula

$$
T v=\epsilon^{d} t r
$$

Then equations (8) and (9) define a link invariant. We need to prove that it is of degree $d$. Let $L$ be a link with $l$ self-intersections, and let $x_{0} \in S B_{n}^{g}$ be such that its closure $\hat{x}_{0}$ is ambient isotopic to $L$. We use here the generalizations of the theorem of J. Alexander and the theorem of A. A. Markov for singular links and braids proved by Joan Birman [6] and Bernd Gemein [10]. Let $x_{1}$ be the image of $x_{0}$ in $V^{g}$. Then

$$
x_{1}=y_{1} a_{i_{1}} y_{2} a_{i_{2}} \ldots a_{i_{l}} y_{l+1},
$$

where the elements $y_{i}$ do not have entries of generators $a_{j}$. Define $x \in K B r^{g}$ by the formula

$$
x=y_{1}\left(\sigma_{i_{1}}-\sigma_{i_{1}}^{-1}\right) y_{2}\left(\sigma_{i_{2}}-\sigma_{i_{2}}^{-1}\right) \ldots\left(\sigma_{i_{l}}-\sigma_{i_{l}}^{-1}\right) y_{l+1} .
$$

Then

$$
\epsilon^{d} \operatorname{tr}(x)=T v(x)=\epsilon^{l} T\left(x_{1}\right) .
$$

Since $\operatorname{tr}(x) \in K$ and $T\left(x_{1}\right) \in K[\epsilon]$, if $l>d$ we must have $\operatorname{tr}(x)=0$. By construction

$$
\mathcal{L}(L)=z^{1-n} \operatorname{tr}(x)
$$

so it follows that $\mathcal{L}(L)=0$.

As an example of the situation considered above let

$$
T_{S}: \mathbb{Z} B r \rightarrow \mathbb{Z}(\epsilon)
$$

be the trace constructed by V. Turaev [13, Theorem 4.2.1]. It is a Markov trace with $z=1$ :

$$
T_{S}\left(x \sigma_{n}^{ \pm 1}\right)=T_{S}(x) \text { for all } x \in B r_{n} \subset B r .
$$

For $i=0, \ldots, g$; we define a trace

$$
T_{S, i}: \mathbb{Z} B r^{g} \rightarrow \mathbb{Z}(\epsilon)
$$

as the composition

$$
\mathbb{Z} B r^{g} \stackrel{\phi}{\longrightarrow} \mathbb{Z} B r^{g-i} \rightarrow \mathbb{Z} B r \stackrel{T_{S}}{\longrightarrow} \mathbb{Z}(\epsilon)
$$


where the first map is defined by the formulas

$$
\begin{gathered}
\phi\left(\tau_{j}\right)=e, \text { for } j=1, \ldots, i ; \\
\phi\left(\tau_{j}\right)=\tau_{j-i}, \text { for } j=i+1, \ldots, g ; \\
\phi\left(\sigma_{k}\right)=\sigma_{k}, \text { for all } k ;
\end{gathered}
$$

and the second one is the canonical inclusion. It follows from the presentations (2) of the groups $\mathrm{Br}_{n}^{g}$ that the homomorphism $\phi$ is defined correctly. Then we apply the standard substitution $q=\exp (\epsilon)$ and obtain the unique Markov trace

$$
T_{i}: V^{g} \rightarrow \mathbb{Z}[[\epsilon]],
$$

such that $T_{i} v^{g}=T_{S, i}$. We write

$$
T_{i}=\sum_{d=0}^{\infty} T_{i, d}
$$

where each

$$
T_{i, d}: V^{g} \rightarrow \mathbb{Z}[\epsilon]
$$

is a Markov trace homogeneous of degree $d$ with

$$
T_{i, d}\left(x \sigma_{n}^{ \pm 1}\right)=T_{i, d}(x) \text { for all } x \in V_{n}^{g} \subset V^{g} .
$$

So, each $T_{i, d}$ gives a link invariant $\mathcal{L}_{i, d}$ of degree $d$, such that

$$
\mathcal{L}_{i, d}(\hat{x})=\epsilon^{-d} T_{i, d}\left(v^{g}(x)\right) \text {, for } x \in B r_{n}^{g} .
$$

\section{REFERENCES}

1. V. A. Vassiliev, Complements of discriminants of smooth maps: topology and applications, (Translations of mathematical monographs; v. 98), AMS, Providence, 1992.

2. Xiao-Song Lin, Finite type link invariants of 3-manifolds, Topology 33 , No 1 (1994), 45-71.

3. V. A. Vassiliev, On invariants and cohomology of spaces of knots in arbitrary manifolds, Preprint, Independent Moscow University, 20 pages (1997).

4. V. V. Goryunov, Vassiliev invariants of knots in a solid torus, Preprint, University of Liverpool (1997), 34 pages.

5. John C. Baez, Link Invariants of Finite Type and Perturbation Theory, Letters in Math. Physics 26 (1992), 43-51.

6. Joan S. Birman, New points of view in knot theory, Bull. of the Amer. Math. Soc. 28 No 2 (1993), 253-387.

7. A. B. Sossinsky, Preparation theorems for isotopy invariants of links in 3-manifolds, in Quantum Groups, Proceedings (Lecture Notes in Math.; No 1510) (1992), Springer-Verlag, Berlin a. o., 354-362.

8. V. V. Vershinin, Homology of Braid Groups in Handlebodies, Preprint No 96/06-2, Université de Nantes (1996).

9. V. V. Vershinin, On Braid Groups in Handlebodies, to appear in Siberian Math. J.

10. Bernd Gemein, Singular Braids and Markov's Theorem, Preprint, University of Düsseldorf, 15 pages, 1996, to appear in J. of Knot Theory and its Ramifications.

11. S. Lambropoulou and C. Rourke, Markov's theorem in 3-manifolds, Topology and Its Appl. 78 (1997).

12. R. Fenn, E. Keyman and C. Rourke, The Singular Braid Monoid Embeds in a Group, Preprint, 1996.

13. V. G. Turaev, The Yang-Baxter equation and invariants of links, Invent. Math. 92 (1988), 527-553.

Sobolev Institute of Mathematics, Novosibirsk, 630090, Russia

E-mail address: versh@math.nsc.ru 